\title{
Malnutrition and cachexia among cancer out-patients in Nairobi, Kenya
}

\author{
L. U. Kaduka ${ }^{1}$, Z. N. Bukania ${ }^{1}$, Y. Opanga ${ }^{2}$, R. Mutisya ${ }^{1}$, A. Korir ${ }^{3}$, V. Thuita ${ }^{4}$, C. Nyongesa ${ }^{5}$, \\ M. Mwangi ${ }^{1}$, C. F. L. Mbakaya ${ }^{6}$ and E. Muniu ${ }^{1}$ \\ ${ }^{1}$ Centre for Public Health Research, Kenya Medical Research Institute, Nairobi, Kenya \\ ${ }^{2}$ School of Public Health, Moi University, Eldoret, Kenya \\ ${ }^{3}$ Centre for Clinical Research, Kenya Medical Research Institute, Nairobi, Kenya \\ ${ }^{4}$ Department of Nutrition, Kenyatta National Hospital, Nairobi, Kenya \\ ${ }^{5}$ Texas Cancer Centre, Nairobi, Kenya \\ ${ }^{6}$ Rongo University, Rongo, Kenya
}

(Received 27 June 2017 - Final revision received 4 September 2017 - Accepted 4 October 2017)

Journal of Nutritional Science (2017), vol. 6, e63, page 1 of 10

doi:10.1017/jns.2017.61

Abstract

Cancer is the third leading cause of death in Kenya. However, there is scarce information on the nutritional status of cancer patients to guide in decision making. The present study sought to assess the risk of malnutrition, and factors associated with malnutrition and cachexia, among cancer out-patients, with the aim of informing nutrition programmes for cancer management in Kenya and beyond. This was a facility-based cross-sectional study performed at Kenyatta National Hospital and Texas Cancer Centre in Nairobi, Kenya. The risk of malnutrition was assessed using the Malnutrition Universal Screening Tool (MUST). Diagnoses of malnutrition and cachexia were done using the European Society of Clinical Nutrition and Metabolism (ESPEN) and Fearon criteria, respectively. A total of 512 participants were assessed. Those at risk of malnutrition were $33.1 \%(12.5 \%$ at medium risk, $20.6 \%$ at high risk). Prevalence of malnutrition was $13.4 \%$. The overall weight loss $>5 \%$ over 3 months was $18.2 \%$ and low fat-free mass index was $43.1 \%$. Prevalence of cachexia was $14.1 \%$ compared with $8.5 \%$ obtained using the local criteria. Only $18.6 \%$ participants had received any form of nutrition services. Age was a predictor of malnutrition and cachexia in addition to site of cancer for malnutrition and cigarette smoking for cachexia. The use of the MUST as a screening tool at the first point of care should be explored. The predictive value of current nutrition assessment tools, and the local diagnostic criteria for malnutrition and cachexia should be reassessed to inform the development of appropriate clinical guidelines and future capacity-building initiatives that will ensure the correct identification of patients at risk for timely care.

Key words: Malnutrition: Cancer: Body composition: Kenya: Sub-Saharan Africa

Cancer is the third leading cause of death in Kenya with a mortality rate estimated at 26941 annually ${ }^{(1)}$. Malnutrition and cachexia are common in cancer and often contribute to poor health outcomes. Malnutrition is a clinical condition characterised by an imbalance of energy, protein and other nutrients that causes measurable adverse effects on body tissue, function and clinical outcome ${ }^{(2)}$. Cancer cachexia is a multifactorial syndrome characterised by an ongoing loss of skeletal muscle mass, with or without loss of fat mass that cannot be fully reversed by conventional nutrition support ${ }^{(3)}$. Cachexia is a manifestation in advanced cancer that affects $50-80 \%$ of cancer patients and accounts for up to $20 \%$ of cancer deaths ${ }^{(4,5)}$. Early detection and management of malnutrition and cachexia are therefore key. This has necessitated the integration of nutrition care in cancer management for improved clinical outcomes and quality of life ${ }^{(6)}$.

Abbreviations: AOR, adjusted OR; ESPEN, European Society of Clinical Nutrition and Metabolism; FFMI, fat-free mass index; FVS, Food Variety Score; KEMRI, Kenya Medical Research Institute; KNH, Kenyatta National Hospital; MUST, Malnutrition Universal Screening Tool; TCC, Texas Cancer Centre.

* Corresponding author: L. U. Kaduka, fax +25422720030, email lydia.kaduka@gmail.com and lkaduka@kemri.org 
The 2013 National Guidelines for Cancer Management in Kenya identifies nutrition care for persons with cancer as an integral part of cancer treatment ${ }^{(7)}$. It singles out the need for early initiation of management plans that incorporate nutritional assessment, diagnosis and intervention. BMI and weight loss are currently the main indicators of malnutrition and cachexia, respectively, in Kenyan public hospitals. However, previous studies have shown the limitations of using BMI as the sole indicator of malnutrition in cancer because it can yield high or normal results in patients with large tumours, ascites or oedema, thereby masking weight loss. Unintentional weight loss is instead recommended ${ }^{(8,9)}$.

The Malnutrition Universal Screening Tool (MUST) is designed to identify adults who are underweight or obese and at risk of malnutrition, and who may benefit from appropriate intervention $^{(2)}$. It uses BMI, weight loss and acute disease effect scores in estimating the overall risk of malnutrition. The European Society of Clinical Nutrition and Metabolism (ESPEN) consensus statement provides a minimum set of criteria for the diagnosis of malnutrition that can be applied independently of clinical setting and aetiology ${ }^{(10)}$. It allows for inclusion of parameters such as fat-free mass where applicable in diagnosing malnutrition. The international consensus on definition and classification of cancer cachexia also provides diagnostic criteria for cachexia that include weight loss or loss of skeletal muscle mass ${ }^{(3)}$. These assessments enrich understanding of the variability in nutritional status, and have the potential to alter cancer treatment paradigms for improved health outcomes ${ }^{(11,12)}$.

There is scarce information on the burden of malnutrition and cachexia among cancer out-patients in Kenya to inform decision making and policy ${ }^{(1)}$. In view of the lack of information and the recent advances in screening and diagnosis of malnutrition and cachexia, this study sought to establish the risk of malnutrition, and factors associated with the occurrence of malnutrition and cachexia, with the ultimate aim of informing nutrition programmes for cancer management in Kenya and beyond.

\section{Research methods}

\section{Study design and population}

This was a facility-based cross-sectional study among cancer out-patients seeking treatment at Kenyatta National Hospital (KNH) and Texas Cancer Centre (TCC) in Nairobi, Kenya. $\mathrm{KNH}$ is the largest public referral hospital in Kenya. The $\mathrm{KNH}$ cancer treatment centre receives more than 22000 cancer patients annually ${ }^{(13)}$. TCC, on the other hand, is a private facility that receives approximately 1092 cancer patients annually ${ }^{(14)}$.

\section{Inclusion criteria}

The study included all out-patients with confirmed stage I-IV cancer disease, aged above 18 years and receiving treatment (radiotherapy, chemotherapy, surgery or hormonal) in the two selected cancer treatment sites.

\section{Sample size estimation and allocation}

Prevalence of malnutrition among cancer patients in Kenya remains unknown. Using the Fischer formula ${ }^{(15)}$, a sample size of 512 participants was calculated factoring in a $10 \%$ nonresponse rate. The calculated sample size was distributed between the two facilities using the square root allocation method to ensure appropriate representation of the two facilities based on the number of patients received per facility per year. A systematic random sampling method was used in recruiting participants until the desired sample size was achieved. This entailed obtaining the total number of patients expected in each facility from the hospital records and selecting a random starting point. The sampling interval was determined by dividing the total number expected by the desired sample size.

\section{Recruitment and training of research assistants}

Research assistants with a medical background were recruited and trained on the protocol, study procedures including the consenting process, study tools and on proper data collection using pretested data collection tools. Training and certification were done to harmonise data collection methods between researchers. This was followed by piloting of the study tools and definitive data collection.

\section{Assessments}

Sociodemographic assessments. Data collection was done between June and July 2016. Interviews were carried out and information on socio-economic and demographic characteristics collected using a pretested structured questionnaire. Education level was categorised into four categories: none, primary (1-8 years), secondary (9-14 years), and tertiary ( $>14$ years). Occupation was classified as formal, non-formal, unemployed and retirees.

Anthropometric assessments. Weight and height were measured using calibrated Seca 762 classic mechanical medical weighing scales and UNICEF standard height boards, and findings recorded to the nearest $0.1 \mathrm{~kg}$ and 0.1 $\mathrm{cm}$, respectively. In measuring height, participants were asked to stand upright, without head gear and shoes, arms hanging loosely at the sides, feet slightly apart, and with the back of the heels and head against the stadiometer. In measuring weight, the scale was placed on a flat surface and zero checked before every measurement. Participants were weighed without shoes while wearing minimal clothing. Weight and height measurements were used to compute BMI using the formula of BMI = weight $(\mathrm{kg}) /$ height $\left(\mathrm{m}^{2}\right)$. Information on weight history was retrieved from patients' medical records and used to calculate changes in weight. The current weight was deducted from the previous weight to calculate the amount of weight lost.

Body composition assessments. and lean mass percentages
Total body fat, body water were estimated using a 
commercially available single-frequency four-electrode bioimpedance analyser system (Bodystat 1500). Tetrapolar hand to foot measurements were performed in a supine position lasting for $15 \mathrm{~min}$. The gel-filled electrodes were placed on the dorsal surfaces of the right hand and foot, distal ones being, respectively, proximal to the metacarpal and metatarsal phalangeal joints in accordance with standard tetrapolar electrode placement to minimise gap impedence ${ }^{(16)}$. The instrument recorded whole body impedance from the hands to the feet by applying an electric alternating current flux of $0.8 \mathrm{~A}$ at an operating frequency of $50 \mathrm{kHz}$. Fat-free mass index (FFMI) was calculated using the formula FFMI $=$ lean body mass $(\mathrm{kg}) /$ height $\left(\mathrm{m}^{2}\right)$.

Clinical and dietary assessments. Details on participants' diagnosis (clinical history, types of cancer and clinical stages) and treatment were retrieved from patient files. Information on symptoms such as lack of appetite, feeling nauseated or vomiting was self-reported. Dietary diversity was assessed using individual Food Variety Scores (FVS) that collect data on specific food items consumed ${ }^{(17)}$. Participants provided details on the variety of foods consumed $7 \mathrm{~d}$ and $24 \mathrm{~h}$ prior to the interview. Each food item was given a score of 1 if consumed at least once over $24 \mathrm{~h}$ and the $7 \mathrm{~d}$ period regardless of the frequency. The FVS were classified as either adequate by creating a cut-off point where the mean of the upper tercile was used to classify the respondent as either having adequate or inadequate dietary diversity.

Risk of malnutrition. Participants were screened using the MUST to identify those at risk of malnutrition. The overall risk was an aggregate of BMI, weight loss and acute disease scores whose cut-off points and interpretation of the data were done in accordance with MUST guidelines ${ }^{(2)}$. BMI cut-offs of $>20,18.5-20$ and $<18.5 \mathrm{~kg} / \mathrm{m}^{2}$ were scored as 0,1 and 2, respectively. Unplanned weight loss of $<5,5-10$ and $>10 \%$ in the past 3-6 months was scored as 0,1 and 2 , respectively. The acute disease effect did not apply to the out-patients and was scored a zero. A score aggregate of 0 , 1 and 2 represented low, medium and high risk of malnutrition, respectively.

Diagnosis of malnutrition. Diagnosis of malnutrition was done in accordance with the ESPEN criteria for the diagnosis of malnutrition ${ }^{(10)}$. The criteria provide for the use of low BMI $\left(<18.5 \mathrm{~kg} / \mathrm{m}^{2}\right)$, or combined finding of unintentional weight loss of $>5 \%$ over 3 months and at least one of either a reduced BMI of $<20 \mathrm{~kg} / \mathrm{m}^{2}$ or $<22 \mathrm{~kg} /$ $\mathrm{m}^{2}$ in subjects younger and older than 70 years, respectively, or a low FFMI of $<15 \mathrm{~kg} / \mathrm{m}^{2}$ and $<17 \mathrm{~kg} / \mathrm{m}^{2}$ in females and males, respectively, to diagnose malnutrition. Similar to ESPEN, the local criterion uses low BMI in diagnosing malnutrition; hence further bivariate and multivariate analyses were done using low BMI.
Diagnosis of cachexia. Diagnosis of cachexia was in accordance with the Fearon criteria for the assessment of cachexia $^{(3)}$. Diagnosis of cancer cachexia was based on BMI $<20 \mathrm{~kg} / \mathrm{m}^{2}$ and any degree of weight loss $>2 \%$. The local definition of cachexia is $>5 \%$ weight loss/month or $>10 \%$ weight loss over 6 months ${ }^{(7)}$.

Information from the MUST will be used to identify patients at risk of malnutrition for targeted intervention. In addition to information on malnutrition based on low BMI, differences in malnutrition based on weight loss and either a reduced age-specific BMI or low sex-specific FFMI diagnostic criteria will help inform clinical practice, the development of guidelines and appropriate care plans for cancer out-patients in Kenya.

Ethical considerations. This study was conducted according to the guidelines laid down in the Declaration of Helsinki and all procedures involving human subjects were approved by the Kenya Medical Research Institute (KEMRI) Scientific and Ethics Review Unit (reference: KEMRI/SERU/CPHR/ 001/3026) and the KNH Ethics and Research Review Committee (registration certificate: P462/07/2015). Written informed consent was obtained from all study subjects.

Data management and statistical analysis. Quantitative data were double-entered using the Microsoft Access application. Clean and validated data were exported to SPSS version 20.0 statistical software (IBM Corp.) for data analysis. Exploratory data analysis techniques were used at the initial stage of analysis to uncover the data distribution characteristics of continuous variables and identify outliers. Descriptive statistics such as proportions and frequency distributions were used to summarise categorical variables and measures of central tendency and dispersion for continuous variables. Pearson's $\chi^{2}$ test or Fisher's exact test, where applicable, was used to assess the relationship between dependent and independent categorical variables. To test for association between independent continuous variables and dependent categorical variables, the unpaired Student's $t$ test for normally distributed continuous variables and the Mann-Whitney $U$ test for non-normally distributed continuous variables were used for dichotomous dependent categorical variable. For dependent categorical variables with more than two categories, one-way ANOVA for normally distributed continuous variables and Kruskal-Wallis one-way ANOVA for non-normally distributed continuous variables were carried out. The threshold for statistical significance was set at $\alpha=0.05$ (two-sided). Post hoc grouping of study participants was done during analysis since this was a comparative study.

\section{Results}

A total of 512 participants were recruited (male: $28.1 \%$; female: $71.9 \%$ ). The mean age was 52 (SD 13.8) years. The majority of the participants $(71.7 \%)$ were aged 40 years and above, $20.3 \%$ were in formal employment, and $9.8 \%$ had 
not received formal education. The top three site cancers were cancer of the breast $(28.7 \%)$, female genital $(22.7 \%)$ and digestive organs $(21.7 \%)$. The majority $(260 ; 52 \%)$ of the patients presented with late-stage cancer (stages III and IV) compared with $213(42.6 \%)$ with early-stage cancer (stages 0 , I and II). Table 1 shows the sociodemographic and clinical characteristics of the study participants.

Breast $(39.7 \%)$ and cervical $(25 \%)$ cancer were the leading cancers in females, and prostate $(24 \%)$ and oesophagus $(24$ $\%)$ in males. Up to $470(91.8 \%)$ participants were on treatment, out of whom $202(43 \%)$ were on one form of treatment (chemotherapy $114(24.3 \%)$, surgery sixteen $(3.4 \%)$, radiotherapy sixty-eight $(14.5 \%)$ and hormonal therapy four $(0.8$ $\%)$ ), while $183(38.9 \%)$ and eighty-five $(18.1 \%)$ were on two and three combined forms of treatments, respectively. Fatigue and poor appetite were reportedly the most common symptoms experienced in the previous $24 \mathrm{~h}(38.3$ and $32.4 \%$ ),

Table 1. Sociodemographic and clinical characteristics of study participants ( $n$ 512)

(Numbers and percentages)

\begin{tabular}{|c|c|c|}
\hline Characteristics & $n$ & $\%$ \\
\hline \multicolumn{3}{|l|}{ Sex } \\
\hline Female & 368 & 71.9 \\
\hline Male & 144 & $28 \cdot 1$ \\
\hline \multicolumn{3}{|l|}{ Age (years) } \\
\hline Mean & \multicolumn{2}{|c|}{$52 \cdot 01$} \\
\hline SD & \multicolumn{2}{|c|}{13.78} \\
\hline$<30$ years & 22 & $4 \cdot 3$ \\
\hline $30-39$ years & 72 & $14 \cdot 1$ \\
\hline $40-49$ years & 133 & $26 \cdot 0$ \\
\hline $50-59$ years & 135 & $26 \cdot 4$ \\
\hline 60 years and above & 150 & $29 \cdot 3$ \\
\hline \multicolumn{3}{|l|}{ Education levels } \\
\hline No formal education & 50 & 9.8 \\
\hline Primary & 195 & 38.1 \\
\hline Secondary & 171 & 33.4 \\
\hline Tertiary & 96 & $18 \cdot 8$ \\
\hline \multicolumn{3}{|l|}{ Occupation } \\
\hline Formal & 104 & $20 \cdot 3$ \\
\hline Non-formal & 198 & 38.7 \\
\hline Unemployed & 170 & 33.2 \\
\hline Retired & 40 & $7 \cdot 8$ \\
\hline \multicolumn{3}{|l|}{ Marital } \\
\hline Married & 378 & 73.8 \\
\hline Single & 75 & $14 \cdot 6$ \\
\hline Other* & 59 & 11.5 \\
\hline \multicolumn{3}{|l|}{ Stage of cancer $(n 500)$} \\
\hline Stage 0 & 4 & 0.8 \\
\hline Stage 1 & 75 & $15 \cdot 0$ \\
\hline Stage 2 & 134 & $26 \cdot 8$ \\
\hline Stage 3 & 141 & $28 \cdot 2$ \\
\hline Stage 4 & 119 & 23.8 \\
\hline Unknown & 27 & $5 \cdot 4$ \\
\hline \multicolumn{3}{|l|}{ Site of cancer } \\
\hline Breast (C50) & 147 & $28 \cdot 7$ \\
\hline Female genital (C51-58) & 116 & $22 \cdot 7$ \\
\hline Digestive organs (C15-26) & 111 & 21.7 \\
\hline Lip, oral cavity and pharynx (C00-14) & 94 & 18.4 \\
\hline Haematopoietic (C81-96) & 9 & 1.8 \\
\hline Bone, cartilage, melanoma (C40-43) & 18 & 3.5 \\
\hline Respiratory organs (C30-39) & 9 & 1.8 \\
\hline Kaposi sarcoma (C46) & 8 & 1.6 \\
\hline
\end{tabular}

* Divorced, widowed or separated. and 1 month at 50 and $51 \%$, respectively. Up to $35 \%$ reported feeling nauseated while $28 \%$ had vomited in the previous 1 month. Only $18.6 \%$ of the participants reported having received nutrition services in the form of nutrition counselling and education or food support during their hospital visits.

\section{Risk of malnutrition}

The risk of malnutrition was assessed in 471 participants. Those found at risk of malnutrition were $33.1 \%(12.5 \%$ at medium risk and $20.6 \%$ at high risk). The risk of malnutrition was significantly higher in males (OR 2.84 (95\% CI 1.87, 4.31); $P<0.001$ ), patients who smoked cigarettes (OR 2.86 (95\% CI 1.76, 4.66); $P<0.001$ ), those with stage II cancer disease (OR 1.97 (95\% CI 1.12, 3.43); $P=0.017)$, and those on radiotherapy treatment (OR 2.35 (95\% CI 1.36, 4.06); $P=0.002)$, as shown in Table 2. Those aged $<30$ years were less likely to be at risk (OR $0.26(95 \%$ CI 0.09 , $0.67) ; P<0.001)$, and so were patients with breast cancer (OR 0.34 (95\% CI 0.18, 0.63); $P=0.001)$.

\section{Prevalence and factors associated with malnutrition}

Malnutrition was assessed in 506 participants. Malnutrition based on BMI $<18.5 \mathrm{~kg} / \mathrm{m}^{2}$ was $13.4 \%$ (males, $24.8 \%$; females, $8.9 \%$ ). Bivariate analysis revealed males to be 3.39 $(95 \%$ CI $2.01,5.73)$ times likely to be malnourished compared with females. Likewise, the odds of having malnutrition were higher among cigarette smokers (3.19 (95\% CI 1.81, 5.60); $P<0.001$ ), alcohol consumers (2.01 (95\% CI 1.20 , 3.38), $P=0.008)$, patients with stage II cancer ( 2.30 (95\% CI 1.02, 5.17); $P=0.044)$, those with cancer in digestive organs $(2.56$ (95\% CI 1.22, 5.35); $P=0.012)$, and those undergoing surgery $(3.26$ (95\% CI $1.35,7.86) ; P=0.002)$ and radiotherapy treatment $(2.37(95 \% \mathrm{CI} 1.19,4.69) ; P=$ 0.002). The opposite was observed in patients less than 30 years of age and those with breast cancer disease, as shown in Table 3.

The overall weight loss $>5 \%$ over 3 months was $18.2 \%$ and low FFMI $43 \cdot 1 \%$. Malnutrition based on the combined findings of unintentional weight loss of $>5 \%$ over 3 months together with age-specific reduced BMI was $8.6 \%$, and with sex-specific low FFMI was $11.5 \%$.

The mean FVS was 14.4 (SD 5.6). BMI was significantly associated with the previous $24 \mathrm{~h}$ and $7 \mathrm{~d}$ FVS at $P<0.001$ and $P<0.003$, respectively. A majority $(97 \%)$ of participants with low BMI reported inadequate dietary diversity.

\section{Prevalence and factors associated with cachexia}

The prevalence of cachexia based on the Fearon criteria was $14.1 \%$. Being male, cigarette smoking, alcohol consumption and radiotherapy treatment were significantly associated with cachexia, as shown in Table 4. Males were $3.99(95 \%$ CI 2.39-6.67; $P<0.001)$ times more likely to have cachexia compared with females. The odds were higher among cigarette smokers (OR 3.95 (95\% CI 2.29, 6.82); $P<0.001$ ), alcohol 
Table 2. Sociodemographic and clinical factors associated with risk of malnutrition (Numbers and percentages; odds ratios and $95 \%$ confidence intervals)

\begin{tabular}{|c|c|c|c|c|c|c|c|}
\hline \multirow[b]{2}{*}{ Characteristic } & \multicolumn{2}{|c|}{ At risk ${ }^{*}$} & \multicolumn{2}{|c|}{ Not at risk ${ }^{*}$} & \multirow[b]{2}{*}{ OR } & \multirow[b]{2}{*}{$95 \% \mathrm{Cl}$} & \multirow[b]{2}{*}{$P$} \\
\hline & $n$ & $\%$ & $n$ & $\%$ & & & \\
\hline Overall & 156 & 33.1 & 315 & $66 \cdot 9$ & & & \\
\hline \multicolumn{8}{|l|}{ Sex } \\
\hline Female & 89 & $57 \cdot 1$ & 249 & 79.0 & \multicolumn{2}{|c|}{ Reference } & \\
\hline Male & 67 & 42.9 & 66 & $21 \cdot 0$ & 2.84 & $1.87,4.31$ & $<0.001$ \\
\hline \multicolumn{8}{|l|}{ Age } \\
\hline$<30$ years & 13 & $8 \cdot 3$ & 8 & 2.5 & 0.26 & $0.09,0.67$ & 0.006 \\
\hline $30-39$ years & 25 & $16 \cdot 0$ & 44 & 14.0 & 0.74 & $0.39,1.37$ & 0.335 \\
\hline $40-49$ years & 35 & $22 \cdot 4$ & 88 & $27 \cdot 9$ & 1.05 & $0.61,1.81$ & 0.848 \\
\hline $50-59$ years & 44 & $28 \cdot 2$ & 82 & $26 \cdot 0$ & 0.78 & $0.46,1.31$ & 0.356 \\
\hline 60 years and above & 39 & $25 \cdot 0$ & 93 & 29.5 & \multicolumn{2}{|c|}{ Reference } & \\
\hline \multicolumn{8}{|l|}{ Cigarette smoking } \\
\hline Yes & 44 & $28 \cdot 2$ & 38 & $12 \cdot 1$ & 2.86 & $1.76,4.66$ & $<0.001$ \\
\hline No & 112 & $71 \cdot 8$ & 277 & 87.9 & \multicolumn{2}{|c|}{ Reference } & \\
\hline \multicolumn{8}{|l|}{ Alcohol consumption } \\
\hline Yes & 61 & $39 \cdot 1$ & 95 & $30 \cdot 2$ & 1.49 & $1 \cdot 00,2 \cdot 22$ & 0.053 \\
\hline No & 95 & $60 \cdot 9$ & 220 & 69.8 & \multicolumn{2}{|c|}{ Reference } & \\
\hline \multicolumn{8}{|l|}{ Stage of cancer } \\
\hline Unknown & 12 & 7.9 & 13 & $4 \cdot 2$ & 0.71 & $0 \cdot 29,1 \cdot 70$ & 0.444 \\
\hline Stage 0 & 0 & 0 & 3 & 1.0 & \multicolumn{2}{|c|}{ UD } & UD \\
\hline Stage 1 & 26 & $17 \cdot 2$ & 43 & 13.9 & 1.08 & $0.58,2.01$ & 0.793 \\
\hline Stage 2 & 31 & 20.5 & 93 & $30 \cdot 1$ & 1.97 & $1.12,3.43$ & 0.017 \\
\hline Stage 3 & 38 & $25 \cdot 2$ & 90 & $29 \cdot 1$ & 1.56 & $0.91,2.66$ & 0.107 \\
\hline Stage 4 & 44 & $29 \cdot 1$ & 67 & $21 \cdot 7$ & & rence & \\
\hline \multicolumn{8}{|l|}{ Site of cancer } \\
\hline Lip, oral cavity and pharynx (C00-14) & 33 & $21 \cdot 2$ & 54 & $17 \cdot 1$ & \multicolumn{2}{|c|}{ Reference } & \\
\hline Digestive organs (C15-26) & 46 & 29.5 & 53 & $16 \cdot 8$ & 1.42 & $0.79,2.55$ & 0.241 \\
\hline Respiratory organs (C30-39) & 3 & 1.9 & 4 & 1.3 & 1.22 & $0.25,5.83$ & 0.797 \\
\hline Bone, cartilage, melanoma (C40-43) & 9 & $5 \cdot 8$ & 7 & $2 \cdot 2$ & $2 \cdot 10$ & $0 \cdot 72,6 \cdot 18$ & 0.176 \\
\hline Breast (C50) & 24 & $15 \cdot 4$ & 116 & $36 \cdot 8$ & 0.34 & $0.18,0.63$ & 0.001 \\
\hline Female genital (C51-58) & 35 & $22 \cdot 4$ & 73 & 23.2 & 0.78 & $0.43,1.42$ & 0.421 \\
\hline Haematopoietic (C81-96) & 5 & $3 \cdot 2$ & 3 & 1.0 & 2.72 & $0.61,12 \cdot 17$ & 0.189 \\
\hline Kaposi sarcoma (C46) & 1 & 0.6 & 5 & 1.6 & 0.32 & $0.03,2.93$ & 0.318 \\
\hline \multicolumn{8}{|l|}{ Type of treatment } \\
\hline Chemotherapy & 95 & 68.8 & 238 & $82 \cdot 1$ & \multicolumn{2}{|c|}{ Reference } & \\
\hline Surgery & 10 & $7 \cdot 2$ & 17 & 5.9 & 1.47 & $0.65,3.33$ & 0.352 \\
\hline Radiotherapy & 31 & $22 \cdot 5$ & 33 & 11.4 & $2 \cdot 35$ & $1.36,4.06$ & 0.002 \\
\hline Hormonal therapy & 1 & 0.3 & 2 & 1.4 & 5.01 & $0.45,55 \cdot 91$ & 0.19 \\
\hline
\end{tabular}

UD, undetermined; MUST, Malnutrition Universal Screening Tool.

${ }^{*}$ Risk of malnutrition assessed using the MUST ((BMl cut-offs of $>20,18.5-20$ and $<18.5 \mathrm{~kg} / \mathrm{m}^{2}$ representing low, medium and high risk, respectively) + (unplanned weight loss of $<5 \%, 5-10 \%$ and $>10 \%$ in the past 3-6 months representing low, medium and high risk, respectively)).

consumers (OR 1.91 (95\% CI 1.15, 3.17); $P=0 \cdot 012)$ and patients on radiotherapy treatment (OR 2.63 (95\% CI 1.39, 4.97); $P=0.003)$. Patients with breast cancer were $0.19(95 \%$ CI $0 \cdot 07,0 \cdot 51, P=0 \cdot 001)$ times more likely to have cachexia compared with those with lip, oral cavity and pharynx cancer. Unlike malnutrition, patients $<30$ years of age were $6.52(95 \%$ CI $2 \cdot 45,17 \cdot 36 ; P<0 \cdot 001)$ times more likely to have cachexia.

Prevalence of cachexia based on the local definition of cachexia (>10\% weight loss over 6 months) was $8.5 \%$.

\section{Predictors of risk of malnutrition, malnutrition and cachexia}

Binary logistic regression using the backward conditional method was performed to examine the effect of independent predictors on the risk of malnutrition, malnutrition and cachexia. Age, cigarette smoking and site of cancer were considered for multivariate analysis. The risk of malnutrition was significantly associated with age $<30$ years (adjusted OR (AOR) $0.21(95 \%$ CI $0.07,0.62) ; P=0.005)$, the 30-39 years age group (AOR 0.41 (95\% CI 0.20, 0.84); $P=0 \cdot 015)$, cigarette smoking (AOR 0.36 (95\% CI 0.20, 0.67); $P=0.001$ ) and bone, cartilage and melanoma cancer (AOR 2.95 (95\% CI $1.42,6 \cdot 12) ; P=0.004)$. Malnutrition was significantly associated with age $<30$ years (AOR 0.12 (95\% CI 0.03, 0.413); $P=0.001$ ), cigarette smoking (AOR 0.31 (95\% CI 0.15 , $0.65) ; P=0.002)$, digestive organ cancer (AOR 6.79 (95\% CI 1.38, 33.34); $P=0.018$ ) and breast cancer (AOR 0.30 (95\% CI $0.13,0.70) ; P=0.005)$. Cachexia was significantly associated with age $<30$ years (AOR 10.71 (95\% CI 3.16, 36.32); $P<0.001)$ and cigarette smoking (AOR 2.72 (95\% CI 1.23, 6.02); $P=0.014)$. Table 5 shows age, cigarette smoking and site of cancer as predictors of risk and presence of malnutrition, and only age and cigarette smoking for cachexia.

\section{Discussion}

The present study sought to establish the prevalence and factors associated with malnutrition and cachexia among cancer 
Table 3. Sociodemographic and clinical factors associated with malnutrition (Numbers and percentages; odds ratios and $95 \%$ confidence intervals)

\begin{tabular}{|c|c|c|c|c|c|c|c|}
\hline \multirow[b]{2}{*}{ Characteristic } & \multicolumn{2}{|c|}{ With malnutrition* } & \multicolumn{2}{|c|}{$\begin{array}{c}\text { Without } \\
\text { malnutrition* }\end{array}$} & \multirow[b]{2}{*}{ OR } & \multirow[b]{2}{*}{$95 \% \mathrm{Cl}$} & \multirow[b]{2}{*}{$P$} \\
\hline & $n$ & $\%$ & $n$ & $\%$ & & & \\
\hline Overall & 68 & $13 \cdot 4$ & 438 & $86 \cdot 6$ & & & \\
\hline \multicolumn{8}{|l|}{ Sex } \\
\hline Female & 32 & $47 \cdot 1$ & 329 & $75 \cdot 1$ & \multicolumn{2}{|r|}{ Reference } & \\
\hline Male & 36 & $52 \cdot 9$ & 109 & 24.9 & 3.39 & $2 \cdot 01,5 \cdot 73$ & $<0.001$ \\
\hline \multicolumn{8}{|l|}{ Age } \\
\hline$<30$ years & 8 & 11.8 & 14 & $3 \cdot 2$ & 0.23 & $0.08,0.63$ & 0.004 \\
\hline $30-39$ years & 9 & $13 \cdot 2$ & 62 & $14 \cdot 2$ & 0.90 & $0.38,2.14$ & 0.812 \\
\hline $40-49$ years & 20 & 29.4 & 112 & $25 \cdot 6$ & 0.73 & $0.37,1.47$ & 0.379 \\
\hline $50-59$ years & 14 & $20 \cdot 6$ & 120 & $27 \cdot 4$ & $1 \cdot 12$ & $0.53,2.37$ & 0.765 \\
\hline 60 years and above & 17 & $25 \cdot 0$ & 130 & 29.4 & \multicolumn{2}{|r|}{ Reference } & \\
\hline \multicolumn{8}{|l|}{ Cigarette smoking } \\
\hline Yes & 24 & $35 \cdot 3$ & 64 & 14.6 & 3.19 & $1.81,5.60$ & $<0.001$ \\
\hline No & 44 & $64 \cdot 7$ & 374 & 85.4 & \multicolumn{2}{|r|}{ Reference } & \\
\hline \multicolumn{8}{|l|}{ Alcohol consumption } \\
\hline Yes & 32 & $47 \cdot 1$ & 134 & $30 \cdot 6$ & $2 \cdot 01$ & $1 \cdot 20,3 \cdot 38$ & 0.008 \\
\hline No & 36 & 52.9 & 304 & 69.4 & \multicolumn{2}{|r|}{ Reference } & \\
\hline \multicolumn{8}{|l|}{ Stage of cancer } \\
\hline Unknown & 4 & $6 \cdot 0$ & 23 & 5.4 & 1.09 & $0.34,3.52$ & 0.882 \\
\hline Stage 0 & 0 & 0 & 4 & 0.9 & \multicolumn{2}{|r|}{ UD } & UD \\
\hline Stage 1 & 12 & 17.9 & 63 & $14 \cdot 8$ & 0.99 & $0.45,2.19$ & 0.995 \\
\hline Stage 2 & 10 & 14.9 & 121 & $28 \cdot 3$ & $2 \cdot 30$ & $1 \cdot 02,5 \cdot 17$ & 0.044 \\
\hline Stage 3 & 22 & $32 \cdot 8$ & 116 & $27 \cdot 2$ & 1.00 & $0.51,1.95$ & 0.996 \\
\hline Stage 4 & 19 & 28.4 & 100 & 23.4 & \multicolumn{2}{|r|}{ Reference } & \\
\hline \multicolumn{8}{|l|}{ Site of cancer } \\
\hline Lip, oral cavity and pharynx (CO0-14) & 12 & $17 \cdot 6$ & 82 & $18 \cdot 7$ & \multicolumn{2}{|r|}{ Reference } & \\
\hline Digestive organs (C15-26) & 30 & $44 \cdot 1$ & 80 & $18 \cdot 3$ & 2.56 & $1 \cdot 22,5 \cdot 35$ & 0.012 \\
\hline Respiratory organs (C30-39) & 2 & 2.9 & 6 & 1.4 & $2 \cdot 28$ & $0.41,12.6$ & 0.346 \\
\hline Bone, cartilage, melanoma (C40-43) & 4 & 5.9 & 142 & $32 \cdot 4$ & 1.95 & $0.55,6.92$ & 0.3 \\
\hline Breast (C50) & 4 & 5.9 & 142 & $32 \cdot 4$ & 0.19 & $0.60,0.62$ & 0.006 \\
\hline Female genital (C51-58) & 13 & $19 \cdot 1$ & 100 & $22 \cdot 8$ & 0.89 & $0.38,2.05$ & 0.782 \\
\hline Haematopoietic (C81-96) & 2 & 2.9 & 7 & 1.6 & 1.95 & $0.36,10.52$ & 0.436 \\
\hline Kaposi sarcoma (C46) & 1 & 1.5 & 7 & 1.6 & 0.97 & $0.11,8.65$ & 0.983 \\
\hline \multicolumn{8}{|l|}{ Type of treatment } \\
\hline Chemotherapy & 36 & 61 & 323 & $80 \cdot 8$ & \multicolumn{2}{|r|}{ Reference } & \\
\hline Surgery & 8 & $13 \cdot 6$ & 22 & 5.5 & 3.26 & $1.35,7.86$ & 0.008 \\
\hline Radiotherapy & 14 & $23 \cdot 7$ & 53 & $13 \cdot 3$ & $2 \cdot 37$ & $1 \cdot 19,4.69$ & 0.013 \\
\hline Hormonal therapy & 1 & 1.7 & 2 & 0.5 & 4.49 & $0.39,5.71$ & 0.225 \\
\hline
\end{tabular}

UD, undetermined; ESPEN, European Society of Clinical Nutrition and Metabolism.

${ }^{*}$ Based on ESPEN diagnostic criterion for malnutrition $\left(B M l<18.5 \mathrm{~kg} / \mathrm{m}^{2}\right)$.

out-patients in Nairobi, Kenya. A third of the patients were found to be at risk of malnutrition, while 13.4 and $14.1 \%$ had malnutrition and cachexia, respectively. Our findings confirm previous results that show malnutrition and cachexia as common nutritional challenges among cancer patients, and that negatively make an impact on the response to cancer treatment, quality of life and overall prognosis ${ }^{(18-21)}$. Malnutrition and cachexia are poor prognostic factors and should be prevented or detected and managed early enough ${ }^{(18,22)}$. Those found at risk of malnutrition should therefore be closely monitored to avert progression to frank malnutrition. Mechanisms should also be put in place to identify patients at risk of cachexia for the initiation of preventive interventions ${ }^{(3)}$.

The leading cancers were breast and cervical cancer in females and prostate and oesophageal cancer in males. Our findings agree with the Global Burden of Disease 2016 report that showed prostate and breast cancer as the leading types of cancer in men and women, respectively ${ }^{(23)}$. Up to $24 \%$ of males and close to $10 \%$ of females presented with oesophageal cancer that has been found to contribute to some of the highest incidences of malnutrition ${ }^{(21)}$. This might partly explain the significant association observed between malnutrition and cancer of digestive organs in the present study.

Up to $28.2 \%$ presented with stage III cancer disease. However, the odds of being at risk and having malnutrition were higher among patients with stage II cancer disease. Previous studies have shown that many cancer patients in Africa are diagnosed with advanced cancer and easily become malnourished ${ }^{(24,25)}$. In light of the present findings on increased odds of malnutrition in early cancer and the fact that $81 \%$ of the patients do not receive nutrition services, there is need for enhanced early screening that can be achieved by integrating nutrition services across the four levels of health care in Kenya.

Many factors can modify nutritional status in cancer patients including nausea, vomiting, decreased energy intake or oncologic treatments resulting in malnutrition ${ }^{(26,27)}$. More than $50 \%$ 
Table 4. Sociodemographic and clinical factors associated with cachexia (Numbers and percentages; odds ratios and $95 \%$ confidence intervals)

\begin{tabular}{|c|c|c|c|c|c|c|c|}
\hline \multirow[b]{2}{*}{ Characteristic } & \multicolumn{2}{|c|}{ With cachexia* } & \multicolumn{2}{|c|}{ Without cachexia* } & \multirow[b]{2}{*}{ OR } & \multirow[b]{2}{*}{$95 \% \mathrm{Cl}$} & \multirow[b]{2}{*}{$P$} \\
\hline & $n$ & $\%$ & $n$ & $\%$ & & & \\
\hline Overall & 72 & $14 \cdot 1$ & 440 & 85.9 & & & \\
\hline \multicolumn{8}{|l|}{ Sex } \\
\hline Female & 32 & $44 \cdot 4$ & 335 & $76 \cdot 1$ & \multicolumn{2}{|c|}{ Reference } & \\
\hline Male & 40 & $55 \cdot 6$ & 105 & 23.9 & 3.99 & $2 \cdot 39,6 \cdot 67$ & $<0.001$ \\
\hline \multicolumn{8}{|l|}{ Age } \\
\hline$<30$ years & 10 & $13 \cdot 9$ & 12 & $2 \cdot 7$ & 6.52 & $2 \cdot 45,17 \cdot 36$ & $<0.001$ \\
\hline $30-39$ years & 9 & $12 \cdot 5$ & 63 & $14 \cdot 3$ & 1.12 & $0.47,2.65$ & 0.8 \\
\hline $40-49$ years & 17 & 23.6 & 116 & $26 \cdot 4$ & 1.15 & $0.56,2.35$ & 0.708 \\
\hline $50-59$ years & 19 & $26 \cdot 4$ & 116 & $26 \cdot 4$ & $1 \cdot 28$ & $0.63,2.58$ & 0.488 \\
\hline 60 years and above & 17 & 23.6 & 133 & $30 \cdot 2$ & \multicolumn{2}{|c|}{ Reference } & \\
\hline \multicolumn{8}{|l|}{ Cigarette smoking } \\
\hline Yes & 28 & 38.9 & 61 & $13 \cdot 9$ & 3.95 & $2 \cdot 29,6 \cdot 82$ & $<0.001$ \\
\hline No & 44 & $61 \cdot 1$ & 379 & $86 \cdot 1$ & \multicolumn{2}{|c|}{ Reference } & \\
\hline \multicolumn{8}{|l|}{ Consuming alcohol } \\
\hline Yes & 33 & $45 \cdot 8$ & 135 & $30 \cdot 7$ & 1.91 & $1 \cdot 15,3 \cdot 17$ & 0.012 \\
\hline No & 39 & $54 \cdot 2$ & 305 & $69 \cdot 3$ & \multicolumn{2}{|c|}{ Reference } & \\
\hline \multicolumn{8}{|l|}{ Stage of cancer } \\
\hline Unknown & 6 & 8.5 & 21 & 4.9 & 1.33 & \multirow[t]{2}{*}{$0.48,3.71$} & 0.581 \\
\hline Stage 0 & 0 & 0.0 & 4 & 0.9 & UD & & 0.999 \\
\hline Stage 1 & 13 & $18 \cdot 3$ & 62 & 14.5 & 0.98 & $0.45,2.09$ & 0.955 \\
\hline Stage 2 & 9 & $12 \cdot 7$ & 125 & $29 \cdot 1$ & 0.34 & $0.15,0.77$ & 0.01 \\
\hline Stage 3 & 22 & $31 \cdot 0$ & 119 & $27 \cdot 7$ & 0.86 & $0.45,1.66$ & 0.659 \\
\hline Stage 4 & 21 & 29.6 & 98 & $22 \cdot 8$ & & rence & \\
\hline \multicolumn{8}{|l|}{ Site of cancer } \\
\hline Lip, oral cavity and pharynx (C00-14) & 17 & $23 \cdot 6$ & 77 & $17 \cdot 5$ & \multicolumn{2}{|c|}{ Reference } & \\
\hline Digestive organs (C15-26) & 27 & 37.5 & 84 & $19 \cdot 1$ & 1.45 & $0.73,2.88$ & 0.28 \\
\hline Respiratory organs (C30-39) & 2 & $2 \cdot 8$ & 7 & 1.6 & 1.29 & $0.24,6.78$ & 0.76 \\
\hline Bone, cartilage, melanoma (C40-43) & 4 & 5.6 & 14 & $3 \cdot 2$ & 1.29 & $0.37,4.42$ & 0.681 \\
\hline Breast (C50) & 6 & 8.3 & 141 & $32 \cdot 0$ & 0.19 & $0.07,0.51$ & 0.001 \\
\hline Female genital (C51-58) & 13 & $18 \cdot 1$ & 103 & 23.4 & 0.57 & $0.26,1.24$ & 0.16 \\
\hline Haematopoietic (C81-96) & 3 & 4.2 & 6 & 1.4 & $2 \cdot 26$ & $0.51,9.97$ & 0.28 \\
\hline Kaposi sarcoma (C46) & 0 & 0.0 & 8 & 1.8 & \multicolumn{2}{|c|}{ UD } & UD \\
\hline \multicolumn{8}{|l|}{ Type of treatment } \\
\hline Chemotherapy & 41 & $63 \cdot 1$ & 323 & $80 \cdot 8$ & \multicolumn{2}{|c|}{ Reference } & \\
\hline Surgery & 6 & $9 \cdot 2$ & 24 & $6 \cdot 0$ & 1.97 & $0 \cdot 76,5 \cdot 10$ & 0.163 \\
\hline Radiotherapy & 17 & $26 \cdot 2$ & 51 & $12 \cdot 8$ & $2 \cdot 63$ & $1.39,4.97$ & 0.003 \\
\hline Hormonal therapy & 1 & 1.5 & 2 & 0.5 & 3.94 & $0.35,44.40$ & 0.267 \\
\hline
\end{tabular}

UD, undetermined.

${ }^{*}$ Diagnosis based on Fearon criteria for assessment of cachexia $\left(\mathrm{BMl}<20 \mathrm{~kg} / \mathrm{m}^{2}\right.$ and any degree of weight loss $>2 \%$ ).

of the patients reported having poor appetite and feeling fatigued, while $35 \%$ felt nauseated and $28 \%$ reported vomiting. Inadequate dietary diversity was associated with low BMI. Previous studies have pointed out alterations that occur in components of energy expenditure that can contribute to malnutrition if not compensated for by increased energy intake ${ }^{(27)}$. Thus, a holistic approach is required to address the physical, social, psychological and nutritional needs of cancer outpatients to reduce the consequences of cancer-associated nutritional decline ${ }^{(27,28)}$.

$\mathrm{BMI}$ is the most common and easily available anthropometric assessment method despite its limitation. The local diagnostic criterion is $\mathrm{BMI}<18 \mathrm{~kg} / \mathrm{m}^{2}$, which is similar to the ESPEN criteria. The low BMI criterion was able to identify a higher proportion of patients with malnutrition compared with the combined finding of unintentional weight loss and either a reduced age-specific BMI or a low sex-specific FFMI criteria also recommended by ESPEN. However, the observed low FFMI of $43.1 \%$ highlights the need to incorporate body composition assessments in nutritional assessments. Body composition is not routinely measured in Kenyan public hospitals despite the morbidity risk associated with increased loss of muscle mass. Given its relevance, assessment of body composition should be supported by building the necessary capacity and infrastructure ${ }^{(29-36)}$. On the other hand, the Fearon criteria for diagnosis of cachexia identified $14.1 \%$ cachexia compared with $8.5 \%$ using the local criteria. There is need therefore to evaluate the current local diagnostic criteria for cachexia while being cognizant of the local clinical practice and health system structures.

In this study, malnutrition and cachexia were significantly associated with treatment type and site of cancer. A previous study in Kenya among cervical cancer patients undergoing radiotherapy projected a 2 -year survival of $<20 \%{ }^{(37)}$. Cancer management therefore requires a multimodal approach, and the Kenyan Government recommends establishment of 'multidisciplinary tumour boards' to consider all aspects of the patients' conditions ${ }^{(7)}$. A multidisciplinary team is required 
Table 5. Predictors of risk of malnutrition, malnutrition and cachexia (Adjusted odds ratios and $95 \%$ confidence intervals)

\begin{tabular}{|c|c|c|c|c|c|c|c|c|c|}
\hline & \multicolumn{3}{|c|}{ Risk of malnutrition* } & \multicolumn{3}{|c|}{ Malnutrition† } & \multicolumn{3}{|c|}{ Cachexiał } \\
\hline & Adjusted OR & $95 \% \mathrm{Cl}$ & $P$ & Adjusted OR & $95 \% \mathrm{Cl}$ & $P$ & Adjusted OR & $95 \% \mathrm{Cl}$ & $P$ \\
\hline \multicolumn{10}{|l|}{ Age } \\
\hline$<30$ years & 0.21 & $0.07,0.62$ & 0.005 & 0.12 & $0.03,0.41$ & 0.001 & $10 \cdot 71$ & $3 \cdot 16,36 \cdot 32$ & $<0.001$ \\
\hline 30-39 years & 0.41 & $0.20,0.84$ & 0.015 & 0.43 & $0.16,1.18$ & 0.101 & 2.58 & $0.98,6.79$ & 0.054 \\
\hline $40-49$ years & 0.78 & $0.41,1.49$ & 0.453 & 0.52 & $0.22,1 \cdot 24$ & 0.140 & $1 \cdot 30$ & $0.55,3.10$ & 0.549 \\
\hline $50-59$ years & 0.58 & $0.31,1.06$ & 0.078 & 0.92 & $0 \cdot 39,2 \cdot 20$ & 0.859 & 1.66 & $0.75,3.67$ & 0.212 \\
\hline 60 years and above & \multicolumn{3}{|c|}{ Reference } & \multicolumn{3}{|c|}{ Reference } & \multicolumn{3}{|c|}{ Reference } \\
\hline \multicolumn{10}{|l|}{ Cigarette smoking } \\
\hline Yes & 0.36 & $0.20,0.67$ & 0.001 & 0.31 & $0.15,0.65$ & 0.002 & $2 \cdot 72$ & $1.23,6.02$ & 0.014 \\
\hline No & \multicolumn{3}{|c|}{ Reference } & \multicolumn{2}{|c|}{ Reference } & & \multicolumn{2}{|c|}{ Reference } & \\
\hline \multicolumn{10}{|l|}{ Site of cancer } \\
\hline Lip, oral cavity and pharynx & 0.73 & $0.38,1.41$ & 0.351 & \multicolumn{3}{|c|}{ Reference } & & & \\
\hline Digestive organs & 0.79 & $0.14,4.42$ & 0.788 & $6 \cdot 79$ & $1 \cdot 38,33 \cdot 34$ & 0.018 & & & \\
\hline Respiratory organs & 0.68 & $0 \cdot 19,2.48$ & 0.558 & 0.33 & $0.05,2.06$ & 0.238 & & & \\
\hline Bone, cartilage, melanoma & 2.95 & $1 \cdot 42,6 \cdot 12$ & 0.004 & 1.06 & $0 \cdot 21,5 \cdot 27$ & 0.942 & & & \\
\hline Breast & 1.04 & $0.52,2 \cdot 10$ & 0.906 & 0.30 & $0.13,0.70$ & 0.005 & & & \\
\hline Female genital & 0.83 & $0.15,4.73$ & 0.838 & 0.72 & $0.26,1.97$ & 0.516 & & & \\
\hline Haematopoietic & 3.83 & $0.38,38 \cdot 84$ & 0.256 & 0.39 & $0.06,2.73$ & 0.345 & & & \\
\hline Kaposi sarcoma & \multicolumn{3}{|c|}{ Reference } & 0.75 & $0.07,7.94$ & 0.808 & & & \\
\hline
\end{tabular}

MUST, Malnutrition Universal Screening Tool; ESPEN, European Society of Clinical Nutrition and Metabolism.

* Assessed using the MUST.

† Based on ESPEN diagnostic criterion of $\mathrm{BMl}<18.5 \mathrm{~kg} / \mathrm{m}^{2}$.

$\ddagger$ Based on Fearon criteria of $\mathrm{BMl}<20 \mathrm{~kg} / \mathrm{m}^{2}$ and any degree of weight loss $>2 \%$.

to consider the implications beyond patients' dietary needs to patients' nutritional and functional state throughout the prolonged course of treatment, and is best started earlier rather than later ${ }^{(20,35,38)}$. The role of nutrition as a therapy that complements basic treatment and improves treatment outcomes needs to be appreciated ${ }^{(22,38,39)}$. Inclusion of nutritionists in mainstream oncology practice has the potential to improve early detection and screening of malnutrition, access to nutrition services and communication for improved patient management ${ }^{(22,40)}$. Awareness and consideration of nutritional issues among oncologists and other related health disciplines are also vital to the success of nutrition support in cancer care $^{(24,36)}$. In settings with limited nutritionists/dietitians, provision of protocols for screening can enable task shifting that has been shown to be effective and affordable in improving access to healthcare ${ }^{(41)}$.

Findings on sociodemographic and behavioural factors add insights to the likely influence of social factors in the management of cancer. Disease-aggravating factors such as cigarette smoking and alcohol consumption were prevalent and significantly associated with the presence of malnutrition. Cigarette smoking was a predictor of cachexia. Public sensitisation on the ill-effects of such risk factors and raising the suspicion index among healthcare workers is important. The Global Burden of Disease 2016 report $^{(23)}$ showed that mortality due to all cancers is largely located on the African continent and expected to increase due to the epidemiological transition. Continued research and surveillance therefore remain critical. Further research is needed to understand the nutrition requirements in various care settings, and explore novel cancer preventive and control strategies ${ }^{(19,38)}$. The emergence of population-based cancer registries in East Africa should be supported, and so should prioritisation of cancer control programmes within the health-care systems ${ }^{(19,42-44)}$.

\section{Conclusions}

Malnutrition and cachexia remain a challenge among cancer out-patients in Nairobi, Kenya, yet the provision of nutritional services remains low. The use of the MUST as a screening tool at the first point of care has the potential to identify patients at risk of malnutrition and who are likely to benefit from appropriate interventions for better health outcomes. The local diagnostic criteria for cachexia should be reassessed to ensure correct identification of patients at risk of cachexia for initiation of appropriate care. There is need for inclusion of body composition assessments in Kenyan public hospitals for refined nutritional assessments that will in turn inform patient management plans. Age is a common predictor of malnutrition and cachexia in addition to site of cancer for malnutrition and cigarette smoking for cachexia. Tailored education and sensitisation campaigns should therefore be encouraged and multidisciplinary partnerships formed to provide holistic cancer care.

\section{Limitations}

The study did not measure inflammatory markers or ascertain the aetiology of malnutrition observed. The contribution of inflammation and other catabolic drivers to the development and progression of cachexia was also not assessed. This was partly due to the design of the study and the choice of outpatients in whom tests for inflammatory markers are not routinely done in the selected study facilities.

\section{Acknowledgements}

The study team acknowledges and appreciates the input of various partners who contributed to the development, 
planning and execution of the study. We would like to express our heartfelt appreciation to experts from the Kenya Medical Research Institute (KEMRI), Kenya Cancer Association and $\mathrm{KNH}$ who participated in the planning and implementation processes. Our earnest gratitude goes to the management of collaborating institutions for providing the necessary administrative, technical and logistical support. We would like to thank the following facility staff for their dedication and support during data collection: Lydia Wataka and Onesmus Kariuki of TCC and Irene Karanja and Carol Wambua of KNH. We also thank the following research assistants for their hard work and commitment to this study: Rodgers Ochieng, Melvin Obuya, Schiller Mbuka, Peter Shigholi, Dorine Njeri, Mercy Chepkirui, Paul Odhacha, Isaac Kisiangani and Fidel Muendo. We wish to thank all the study participants and caregivers for their cooperation.

This work was supported by KEMRI (grant no. IRG 176/6) and the Coca-Cola Company (grant no. E-1176). KEMRI and the Coca-Cola Company had no role in the design, analysis or writing of this article.

L. U. K., Z. N. B., Y. O., R. M. and C. F. L. M. contributed to study conceptualisation, design, data collection, analysis and manuscript writing. E. M. and M. M. contributed to the study design, data processing, data analysis and manuscript writing. A. K. participated in study conceptualisation, data analysis and manuscript writing. V. T. and C. N. participated in data collection and manuscript writing.

There were no conflicts of interest.

\section{References}

1. Ministry of Health (2015) Kenya Stepwise Survey for Non Communicable Diseases Risk Factors Report. Nairobi, Kenya: Office of the Director of Medical Services.

2. Todorovic V, Russell C \& Elia M (2011) The 'MUST' Explanatory Booklet. A Guide to the 'Malnutrition Universal Screening Tool' ('MUST') for Adults. Redditch: BAPEN.

3. Fearon K, Strasser F, Anker SD, et al. (2011) Definition and classification of cancer cachexia: an international consensus. Lancet Oncol 12, 489-495.

4. Argilés JM, Busquets S, Stemmler B, et al. (2014) Cancer cachexia: understanding the molecular basis. Nat Rev Cancer 14, 754-762.

5. Meriggi F (2015) Cancer cachexia: one step ahead. Rev Recent Clin Trials 10, 246-250.

6. Meyenfieldt MV (2005) The role of malnutrition in the management of cancer patients - what health professionals need to know; cancer associated malnutrition: an introduction. Eur J Oncol 9, 35-38.

7. Ministry of Health (2013) National Guidelines for Cancer Management in Kenya. Nairobi, Kenya: Office of the Director of Medical Services.

8. Santarpia L, Contaldo F \& Pasanisi F (2011) Nutritional screening and early treatment of malnutrition in cancer patients. J Cachexia Sarcopenia Muscle 2, 27-35.

9. Laky B, Janda M, Cleghorn G, et al. (2008) Comparison of different nutritional assessments and body composition measurements in detecting malnutrition among gynecologic cancer patients. Am J Clin Nutr 87, 1678-1685.

10. Cederholm T, Bosaeus I, Barazzoni R, et al. (2015) Diagnostic criteria for malnutrition - an ESPEN consensus statement. Clin Nutr 34, 335-340.

11. Prado CM, Maia YL, Ormsbee M, et al. (2013) Assessment of nutritional status in cancer - the relationship between body composition and pharmacokinetics. Anticancer Agents Med Chem 13, 1197-1203.
12. Prado CM, Cushen SJ, Orsso CE, et al. (2016) Sarcopenia and cachexia in the era of obesity: clinical and nutritional impact. Proc Nutr Soc 75, 188-198.

13. Muthike C (2013) Nutrition knowledge in association with dietary practices of cancer patients: a case study of Kenyatta National Hospital Cancer Treatment, Nairobi. PhD Thesis, University of Nairobi.

14. Nyongesa C (2014) Texas Cancer Centre Kenya. Article in Construction Review Online. 30 May 2014, Nairobi, Kenya. https://constructionreviewonline.com/2014/05/texas-cancer-centre-kenya/ (accessed November 2017).

15. Fisher RA (1954) Statistical Methods for Research Workers. Edinburgh: Oliver and Boyd.

16. Khalil SF, Mohktar MS \& Ibrahim F (2014) The theory and fundamentals of bioimpedance analysis in clinical status monitoring and diagnosis of diseases. Sensors 14, 10895-10928.

17. Torheim LE, Barikmo I, Parr CL, et al. (2003) Validation of food variety as an indicator of diet quality assessed with a food frequency questionnaire for Western Mali. Eur J Clin Nutr 57, 1283-1291.

18. Irungu CW, Oburra HO \& Ochola B (2015) Prevalence and predictors of malnutrition in nasopharyngeal carcinoma. Clin Med Insights Ear Nose Throat 8, 19-22.

19. Caccialanza R, Cereda E \& Pinto C (2016) Awareness and consideration of malnutrition among oncologists: insights from an exploratory survey. Nutrition 32, 1028-1032.

20. McCreery E \& Costello J (2013) Providing nutritional support for patients with cancer cachexia. Int J Palliat Nurs 19, 32-37.

21. Riccardi D \& Allen K (1999) Nutritional management of patients with esophageal and esophagogastric junction cancer. Cancer Control 6, 64-72.

22. Davies M (2005) Nutritional screening and assessment in cancerassociated malnutrition. Eur J Oncol Nurs 9, Suppl. 2, S64-S73.

23. Fitzmaurice C, Allen C, Barber RM, et al. (2017) Global, regional, and national cancer incidence, mortality, years of life lost, years lived with disability, and disability-adjusted life-years for 32 cancer groups, 1990 to 2015: a systematic analysis for the Global Burden of Disease Study. JAMA Oncol 3, 524-528.

24. De Waele E, Mattens S, Honore PM, et al. (2015) Nutrition therapy in cachectic cancer patients. The Tight Caloric Control ( $\mathrm{TiCaCo})$ pilot trial. Appetite 91, 298-301.

25. Tesfamariam AA, Gebremichael A \& Mufunda J (2013) Breast cancer clinicopathological presentation, gravity and challenges in Eritrea, East Africa: management practice in a resource-poor setting. $S$ Afr Med J 103, 526-528.

26. Mercadante S (1996) Nutrition in cancer patients. Support Care Cancer 4, 10-20.

27. Bosaeus I (2008) Nutritional support in multimodal therapy for cancer cachexia. Support Care Cancer 16, 447-451.

28. Prasad KN (2004) Multiple dietary antioxidants enhance the efficacy of standard and experimental cancer therapies and decrease their toxicity. Integr Cancer Ther 3, 310-322.

29. Andreoli A, De Lorenzo A, Cadeddu F, et al. (2011) New trends in nutritional status assessment of cancer patients. Eur Rev Med Pharmacol Sci 15, 469-480.

30. Valenzuela-Landaeta K, Rojas P \& Basfi-fer K (2012) Nutritional assessment for cancer patient. Nutr Hosp 27, 516-523.

31. Norman K, Stobäus N, Pirlich M, et al. (2012) Bioelectrical phase angle and impedance vector analysis - clinical relevance and applicability of impedance parameters. Clin Nutr 31, 854-861.

32. Caccialanza R, Cereda E, Klersy C, et al. (2015) Phase angle and handgrip strength are sensitive early markers of energy intake in hypophagic, non-surgical patients at nutritional risk, with contraindications to enteral nutrition. Nutrients 7, 1828-1840.

33. Grundmann O, Yoon SL \& Williams JJ (2015) The value of bioelectrical impedance analysis and phase angle in the evaluation of malnutrition and quality of life in cancer patients - a comprehensive review. Eur J Clin Nutr 69, 1290-1297.

34. Crowe PJ, Snyman AM, Dent DM, et al. (1992) Assessing malnutrition in gastric carcinoma: bioelectrical impedance or clinical impression? Aust N Z J Surg 62, 390-393. 
35. Aapro M, Arends J, Bozzetti F, et al. (2014) Early recognition of malnutrition and cachexia in the cancer patient: a position paper of a European School of Oncology Task Force. Ann Oncol 25, 1492-1499.

36. Barrera S \& Demark-Wahnefried W (2009) Nutrition during and after cancer therapy. Oncology (Williston Park) 23, 2 Suppl. Nurse Ed, 15-21.

37. Maranga IO, Hampson L, Oliver AW, et al. (2013) Analysis of factors contributing to the low survival of cervical cancer patients undergoing radiotherapy in Kenya. PLOS ONE 8, e78411.

38. Kim JM \& Sung MK (2016) The efficacy of oral nutritional intervention in malnourished cancer patients: a systemic review. Clin Nutr Res 5, 219-236.

39. Celaya Perez S \& Valero Zanuy MA (1999) Nutritional management of oncologic patients. Nutr Hosp 14, Suppl. 2, 43S-52S.
40. Wood K (2005) Audit of nutritional guidelines for head and neck cancer patients undergoing radiotherapy. J Hum Nutr Diet 18, 343-351.

41. Joshi R, Alim M, Kengne AP, et al. (2014) Task shifting for noncommunicable disease management in low and middle income countries - a systematic review. PLOS ONE 9, e103754.

42. Kantelhardt EJ, Cubasch H \& Hanson C (2015) Taking on breast cancer in East Africa: global challenges in breast cancer. Curr Opin Obstet Gynecol 27, 108-114.

43. Anderson BO, Cazap E, El Saghir NS, et al. (2011) Optimisation of breast cancer management in low-resource and middle-resource countries: executive summary of the Breast Health Global Initiative consensus, 2010. Lancet Oncol 12, 387-398.

44. Lebret T, Coloby P, Descotes JL, et al. (2010) Educational tool-kit on diet and exercise: survey of prostate cancer patients about to receive androgen deprivation therapy. Urology 76, 1434-1439. 